\title{
Kieferorthopädie und gingivale Rezessionen
}

\section{Orthodontics and Gingival Recessions}

\author{
Autor \\ K. Bert $\mathrm{l}^{1,2}$ \\ Institute \\ Abteilung für Parodontologie, Universität Malmö, Schweden \\ 2 Fachbereich für Orale Chirurgie, Universitätszahnklinik Wien, Österreich
}

\author{
Schlüsselwörter \\ - Kieferorthopädie \\ gingivale Rezession \\ - Rezessionsdeckung \\ - Weichgewebsaugmentation \\ Key words \\ orthodontic treatment \\ gingival tissue recession \\ - root coverage procedure \\ - soft tissue augmentation
}

Bibliografie

DOI http://dx.doi.org/

10.1055/s-0035-1549950

Inf Orthod Kieferorthop 2015;

47: 86-92

(c) Georg Thieme Verlag KG

Stuttgart · New York

ISSN 0020-0336

Korrespondenzadresse

PD Dr. Kristina Bertl, MSc.

Abteilung für Parodontologie

Universität Malmö

Carl Gustafs väg 34

SE-20506 Malmö

Schweden

kristina.bertl@mah.se

\section{Zusammenfassung \\ $\nabla$}

Zielsetzung: Anhand folgender 3 Fragestellungen wird ein Überblick zu der Thematik "Kieferorthopädie und gingivale Rezessionen" gegeben:

(1) Stellt eine kieferorthopädische Behandlung einen Risikofaktor für gingivale Rezessionen dar? (2) Kann gingivalen Rezessionen durch eine „prophylaktische“ Weichgewebsaugmentation vor kieferorthopädischer Therapie vorgebeugt werden? (3) Soll die Deckung einer bereits bestehenden gingivalen Rezession vor oder nach der kieferorthopädischen Behandlung erfolgen?

Ergebnis: Eine kieferorthopädische Behandlung als Risikofaktor für gingivale Rezessionen wird nach wie vor kontrovers diskutiert. Jedoch wiesen rezente Studien einen signifikanten Anstieg sowie eine gegenüber der Kontrollgruppe erhöhte Prävalenz an gingivalen Rezessionen in der Retentionsphase nach kieferorthopädischer Behandlung auf. Aber weder die Frage nach der Notwendigkeit einer „prophylaktischen“ Weichgewebsaugmentation vor einer kieferorthopädischen Behandlung noch die Frage nach dem optimalen Zeitpunkt zur Deckung einer bereits bestehenden gingivalen Rezession ist anhand der Literatur eindeutig zu beantworten.

Schlussfolgerung: Trotz noch kontroverser Diskussion scheint eine kieferorthopädische Behandlung als einer von mehreren Faktoren bei der Entstehung gingivaler Rezessionen eine Rolle zu spielen. Dementsprechend sollten andere Risikofaktoren mit berücksichtigt und eine Zahnbewegung aus dem Alveolarfach hinaus möglichst vermieden werden. Die Entscheidung, ob und wann eine kombinierte parodontologisch-kieferorthopädische Behandlung erfolgen sollte, ist nach wie vor subjektiv und nicht evidenz-basiert zu treffen.

\section{Abstract \\ $\nabla$}

Objective: Based on the following 3 questions an overview is given on the topic "orthodontic treatment and gingival tissue recessions":

(1) Should orthodontic treatment be considered as risk factor for developing gingival tissue recessions? (2) Is a "prophylactic" soft tissue augmentation before orthodontic treatment justified to prevent gingival tissue recessions? (3) Should a root coverage procedure of a gingival tissue recession be performed before or after orthodontic treatment?

Results: Orthodontic treatment as a risk factor for gingival tissue recessions is still controversially discussed. Yet, recent studies presented a significant increase as well as compared to a control group a significantly higher prevalence of gingival tissue recessions in the retention phase after orthodontic treatment. Neither the question on the necessity of a "prophylactic" soft tissue augmentation before orthodontic treatment, nor the question on the best time point of root coverage procedures of an already existing gingival tissue recession can be answered by the available literature.

Conclusion: Although this topic is still controversially discussed, orthodontic treatment seems to be an influencing factor in the multifactorial process of gingival tissue recession development. Hence, other known risk factors should be considered and tooth movement out of the alveolar socket should be avoided as far as possible. The decision on any combined periodontal-orthodontic treatment is still made on a subjective and not evidence-based level. 


\section{Einleitung}

$\nabla$

Die Prävalenz gingivaler Rezessionen steigt bereits im jungen Alter deutlich an; Rezessionen über $3 \mathrm{~mm}$ wiesen eine Prävalenz von 6, 24 und 54\% in den Altersgruppen 14-19, 20-29 und 3039 Jahren auf. Dies entspricht einem fast 10 -fachen Anstieg innerhalb von 20 Jahren [1]. Neben einem erhöhten Risiko für Karies und Hypersensibilität am freiliegenden Zahnhals, stellt eine gingivale Rezession in erster Linie eine ästhetische Beeinträchtigung für den Patienten dar. Die Ätiologie wird als multifaktoriell angesehen und zu den Risikofaktoren zählen bspw. eine traumatische Putztechnik, ein dünner gingivaler Phänotyp oder das Tragen eines Lippenpiercings [2]. Aber auch eine kieferorthopädische Behandlung wird als beeinflussender Faktor diskutiert; als mögliche Ursachen für das Entstehen einer gingivalen Rezession während einer kieferorthopädischen Behandlung werden unter anderem eine körperliche Bewegung des Zahnes aus dem Alveolarfach hinaus, ein Ausdünnen des bukkalen Gewebes durch Proklination des Zahnes und/oder eine anhaltende Entzündung aufgrund vermehrter Plaqueanlagerung diskutiert. Da die Hauptmotivation für eine kieferorthopädische Behandlung die ästhetischen Ansprüche des Patienten sind [3], sollten die als zumeist unästhetisch empfundenen gingivalen Rezessionen möglichst vermieden werden. Dementsprechend stellt sich die Frage, ob gingivale Rezessionen tatsächlich durch eine kieferorthopädische Therapie verursacht werden können, und wenn ja, ob man diesen durch eine kombinierte parodontologisch-kieferorthopädische Behandlung vorbeugen kann.

Anhand folgender 3 Fragestellungen soll ein Überblick zu dieser Thematik gegeben werden:

1. Ist eine kieferorthopädische Behandlung ein Risikofaktor für das Entstehen einer gingivalen Rezession?

2. Ist eine „prophylaktische“ Weichgewebsaugmentation zur Vorbeugung gingivaler Rezessionen vor Beginn der kieferorthopädischen Behandlung gerechtfertigt?

3. Soll eine bereits bestehende gingivale Rezession vor oder nach einer kieferorthopädischen Behandlung therapiert werden?

Ist eine kieferorthopädische Behandlung ein Risikofaktor für das Entstehen einer gingivalen Rezession?

Die Neigung der Bezahnung (Pro- oder Retroinklination) stellt hier einen häufig diskutierten Punkt dar. Eine Proklination der Zähne kann zu einem Ausdünnen des bukkalen Hart- und Weichgewebes führen, was in weiterer Folge als Locus minoris resistentiae gilt [4]. Einerseits wurden vermehrt gingivale Rezessionen bei Retroinklination der Unterkieferinzisivi [5] und bei einer finalen Inklination $>95^{\circ}$ in Kombination mit einem dünnen marginalen Gingivarand $(<0.5 \mathrm{~mm})$ beschrieben [6]. Andererseits zeigte sich 5 Jahre nach kieferorthopädischer Behandlung kein signifikanter Unterschied in der Prävalenz gingivaler Rezessionen zwischen proklinierten und nicht proklinierten Unterkieferinzisivi [7] sowie zwischen retroinklinierter, proklinierter und stabiler Zahnstellung [8]. In der letzteren Studie wies die Gruppe mit proklinierter Zahnstellung aber eine 4bzw. 2-fach erhöhte Prävalenz gegenüber den Gruppen mit stabiler und retroinklinierter Zahnstellung auf. In einer aktuellen systematischen Übersichtsarbeit [9] wurde die höhere Proklination der Inzisivi als vermutlich relevanter Risikofaktor für das Entstehen gingivaler Rezessionen bestätigt, jedoch erschien das Ausmaß des Effektes von fraglicher klinischer Relevanz. Des
Weiteren wurde die körperliche Bewegung des Zahnes aus dem Alveolarfach hinaus als Einflussfaktor zur Entstehung gingivaler Rezessionen angeführt. Kein eindeutiger Zusammenhang zeigte sich mit der Behandlungsdauer oder -art, skelettaler und dentaler Beziehung, Alter und Geschlecht der Patienten.

Aufgrund der zumeist multifaktoriellen Ätiologie gingivaler Rezessionen wird auch beim gleichzeitigen Vorliegen anderer „klassischer“ Risikofaktoren ein erhöhtes Risiko für das Entstehen gingivaler Rezessionen während einer kieferorthopädischen Behandlung vermutet $[9,10]$. Zu diesen „klassischen“ Risikofaktoren zählen: gingivale Entzündung, dünner marginaler Gingivarand, dünner gingivaler Phänotyp, schmales Band an keratinisierter Gingiva, bereits bestehende knöcherne Dehiszenz, hohe Plaquewerte, traumatische Putztechnik, Rauchen, hoch ansetzende Lippen- und Wangenbändchen, Tragen eines Lippenpiercings und schmale Kinnsymphyse [2].

Das Fehlen von prospektiven Studien, die Qualität der erhobenen Parameter (z.B. Evaluation der gingivalen Rezessionen nur an Gipsmodellen und keine klinischen Messungen) und eine kurze Nachverfolgungszeit nach Abschluss der kieferorthopädischen Behandlung erklären teilweise die anhaltend kontroverse Diskussion zu dieser Thematik. Rezente, wenn auch nach wie vor retrospektive Studien, die nach den oben angeführten Übersichtsarbeiten $[9,10]$ durchgeführt wurden, präsentieren erstmals Langzeitdaten. Es zeigte sich ein konstanter Anstieg der Prävalenz gingivaler Rezessionen von 7 zu 20 und 38\% vom kieferorthopädischen Behandlungsende zu 2 und 5 Jahren danach. Des Weiteren wurde ein signifikanter Einfluss durch das Alter des Patienten bei Behandlungsende festgestellt: Bei Patienten mit dem Behandlungsende vor dem 16. Lebensjahr traten signifikant weniger gingivale Rezessionen auf. Das Risiko für eine gingivale Rezession stieg mit jedem weiteren Jahr um fast $10 \%$ an. 5 Jahre nach Abschluss der Kieferorthopädie waren die Oberkieferprämolaren am häufigsten betroffen, gefolgt von den Oberkiefereckzähnen, Unterkieferprämolaren, mittleren Unterkieferinzisivi und Oberkiefermolaren. Die Angle Klasse, das Geschlecht, eine durchgeführte Extraktionstherapie und die Art des Retainers zeigten hingegen keinen Zusammenhang mit der Prävalenz gingivaler Rezessionen [11]. In einer weiteren retrospektiven Fall-Kontrollstudie war das Risiko für eine gingivale Rezession in der Retentionsphase nach kieferorthopädischer Behandlung 4,5-fach gegenüber der Kontrollgruppe erhöht (Nachverfolgungszeit bis zu 6 Jahre nach kieferorthopädischem Behandlungsende). Die Prävalenz nahm abermals mit dem Alter zu und am meisten betroffen zeigten sich hier die Unterkieferinzisivi [12].

\section{Zusammenfassung}

- Das Entstehen gingivaler Rezessionen ist in der Regel multifaktoriell bedingt.

- Trotz anhaltender kontroverser Diskussion wurde zuletzt vermehrt ein signifikanter Anstieg der Prävalenz gingivaler Rezessionen in der Retentionsphase nach kieferorthopädischer Behandlung gezeigt.

- Das gleichzeitige Vorliegen „klassischer“ Risikofaktoren scheint zu einem erhöhten Risiko zu führen.

- Zu den „klassischen“ Risikofaktoren zählen: gingivale Entzündung, dünner marginaler Gingivarand, dünner gingivaler Phänotyp, schmales Band an keratinisierter Gingiva, bereits bestehende knöcherne Dehiszenz, hohe Plaquewerte, traumatische Putztechnik, Rauchen, hoch ansetzende Lippen- 
und Wangenbändchen, Tragen eines Lippenpiercings, schmale Kinnsymphyse.

- Zu den „kieferorthopädischen“ Risikofaktoren zählen: körperliche Bewegung des Zahnes aus dem Alveolarfach, Ausdünnen des bukkalen Gewebes durch Proklination, Alter des Patienten, anhaltende Entzündung aufgrund vermehrter Plaqueanlagerung.

- Prospektive Studien mit einem langen Follow-up (zumindest 5 Jahre) inklusive der Aufzeichnung klinischer Parameter, des gingivalen Phänotyps sowie der Mundhygiene vor, während und nach der kieferorthopädischen Behandlung sind notwendig.

Ist eine „prophylaktische“ Weichgewebsaugmentation zur Vorbeugung gingivaler Rezessionen vor Beginn der kieferorthopädischen Behandlung gerechtfertigt?

Trotz nicht eindeutiger Datenlage zur Kieferorthopädie als Risikofaktor für das Entstehen gingivaler Rezessionen wird zur Prävention derselben eine Augmentation des Weichgewebes in der bukko-lingualen Dimension vor kieferorthopädischer Behandlung diskutiert. Einerseits kann diese Therapieoption als empfehlenswert angesehen werden, da ein ausreichend dicker marginaler Gingivarand vermutlich resistenter gegenüber einer Proklination ist [4]. Andererseits kann es aber auch als eine Überbehandlung beurteilt werden, da eine nur möglicherweise entstehende gingivale Rezession auch nach der kieferorthopädischen Behandlung therapiert werden kann. Letztere Ansicht wird unterstützt, da gingivale Rezessionen nach kieferorthopädischer Behandlung größtenteils sehr gut therapierbare Miller-Klasse-I und -II-Rezessionen sind [13] ( $\bullet$ Abb. 1). Andererseits könnte eine dünnere Gewebesituation nach der kieferorthopädischen Behandlung wiederum mit einer verringerten Prognose bei einer Rezessionsdeckung einhergehen; der prozentuale Anteil an vollständiger Deckung ist bei Patienten mit einer dünnen gegenüber einer dicken Weichgewebssituation verringert $[14,15]$.

Die möglichen Indikationsstellungen und der optimale Zeitpunkt für eine Weichgewebsaugmentation zur Vorbeugung gingivaler Rezessionen während einer kieferorthopädischen Behandlung wurden kürzlich von Kloukos und Kollegen zusammengefasst [16]. Jedoch fanden sich auch zu dieser Thematik keine hochqualitativen Studien; lediglich 2 Studien von mittlerer und geringer Qualität $[17,18]$. Beide Studien (je vor über 20 Jahren durchgeführt) führten eine Weichgewebsaugmentation mit einem freien Schleimhauttransplantat vor der kieferorthopädischen Therapie durch. Aufgrund dieser Datenlage kann keine Aussage zum optimalen Zeitpunkt für eine „prophylaktische“ Weichgewebsaugmentation getroffen werden. Darüber hinaus ist das freie Schleimhauttransplantat zur Weichgewebsaugmentation heutzutage größtenteils durch das Bindegewebstransplantat abgelöst worden $[19,20]$. Ein weiterer Schwachpunkt beider Studien ist die Nachverfolgungszeit; die letzte Evaluation erfolgte jeweils am Ende der kieferorthopädischen Behandlung ohne weitere Kontrollen in der Retentionsphase. Auch wenn somit zu einer „prophylaktischen“ Weichgewebsaugmentation im Rahmen einer kieferorthopädischen Behandlung keine eindeutigen Richtlinien gegeben werden können, wird beim gleichzeitigen Vorliegen „klassischer“ Risikofaktoren für das Entstehen gingivaler Rezessionen ein Vorteil für den Patienten durch die Weichgewebsaugmentation vermutet [16]. Daher sollten diese „klassischen“ Risikofaktoren für die Entstehung gingivaler Rezessionen stets berücksichtigt werden - vor und während der kieferorthopädischen Behandlung. Im Speziellen sind hier ein dünner gingivaler Phänotyp, eine bereits bestehende knöcherne Dehiszenz und ein unter $2 \mathrm{~mm}$ breites Band an keratinisierter Gingiva anzuführen $[21,22]$ ( $\bullet$ Abb. 2). Im Rahmen der kieferorthopädischen Planung sollte eine Zahnbewe-
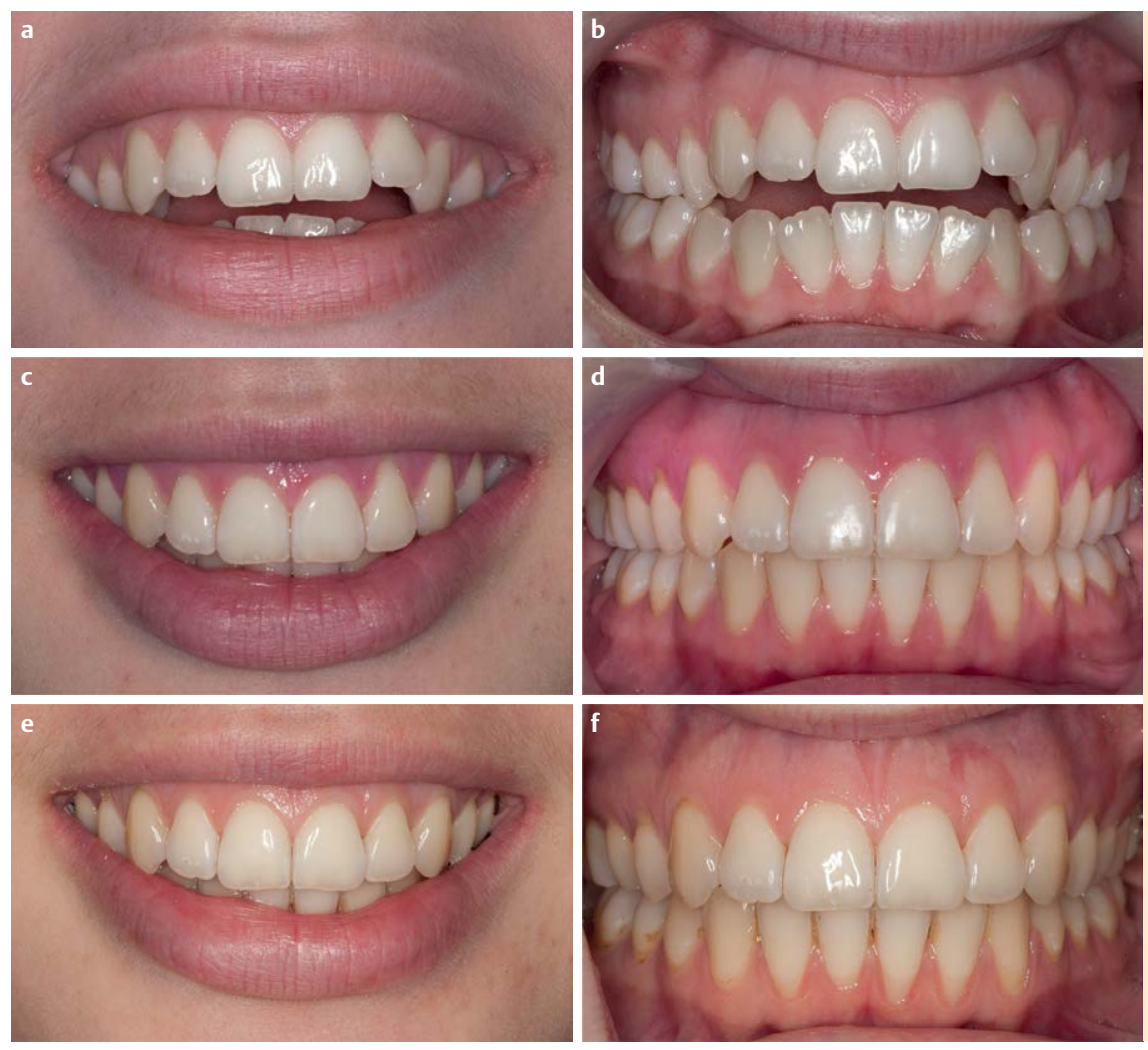

Abb. 1 Patientin vor a, b und nach c, d Kieferorthopädie. Mitunter aufgrund der hohen Lachlinie („Gummy Smile“) bestand bei der Patientin aus ästhetischen Gründen der Wunsch zur Korrektur der gingivalen Rezessionen an Zahn 22 und 24 (je $2 \mathrm{~mm}$ und Miller-Klasse I). e, f Stabile Situation 3 Jahre nach Rezessionsdeckung (koronaler Verschiebelappen mit Applikation von Schmelzmatrixproteinen). (Kieferorthopädie: Dr. Andrea Foltin, Fachbereich für Kieferorthopädie, Universitätszahnklinik Wien). 

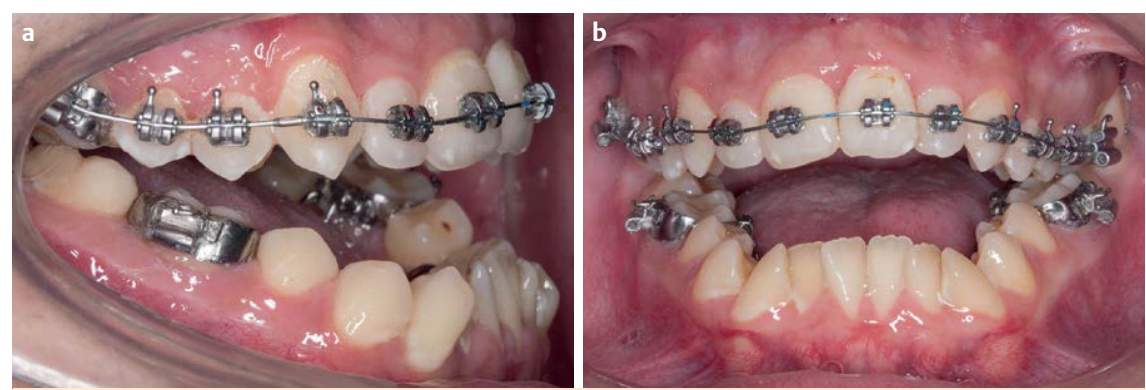

Abb. 2 a Patient mit Angle-Klasse III am Beginn der kieferorthopädischen Behandlung. Vor Dekompensation der Unterkieferfront (vor orthognather Chirurgie) ist eine Weichgewebsaugmentation im Bereich 31 und 41 geplant. b 31 und 41 präsentieren sich mit einem dünnen marginalen Gingivarand, einem minimalen Angebot an keratinisierter Gingiva $(<1 \mathrm{~mm})$ und einer verbesserungswürdigen Mundhygiene. Neben diesen "klassischen" Risikofaktoren, gilt eine präoperative Dekompensation einer Angle-Klasse III vor geplanter orthognather Chirurgie als "kieferorthopädischer" Risikofaktor [22, 25]. (Kieferorthopädie: Dr. Michael Bertl, Fachbereich für Kieferorthopädie, Universitätszahnklinik Wien).

gung aus dem Alveolarfach hinaus und/oder eine übermäßige Expansion des Zahnbogens vermieden werden und Alternativen, wie eine approximale Schmelzreduktion oder eine Extraktionstherapie, in Betracht gezogen werden. Ist eine Expansion des Zahnbogens oder eine Proklination trotz vorliegender anderer Risikofaktoren nicht vermeidbar, erscheint die Weichgewebsaugmentation vor Beginn der Kieferorthopädie als eine mögliche Behandlungsoption. Der Therapieentscheid muss aber subjektiv und aufgrund der zu geringen Datenlage nicht evidenz-basiert getroffen werden.

\section{Zusammenfassung}

- Die Studienlage ist nicht ausreichend, um zu beurteilen, ob eine Weichgewebsaugmentation vor Kieferorthopädie gingivalen Rezessionen vorbeugen kann; der Therapieentscheid bleibt somit subjektiv und nicht evidenz-basiert zu treffen.

- Eine Weichgewebsaugmentation vor Kieferorthopädie kann bei zusätzlichen Risikofaktoren, einer nicht vermeidbaren Expansion des Zahnbogens und/oder Proklination der Zähne in Betracht gezogen werden.

- Randomisierte, kontrollierte Studien sind zu dieser Thematik notwendig.

Soll eine bereits bestehende gingivale Rezession vor oder nach einer kieferorthopädischen Behandlung therapiert werden?

Weist ein Patient (mit oder ohne Wunsch nach einer kieferorthopädischen Behandlung) eine gingivale Rezession auf, stellt sich zunächst die Frage nach der Ätiologie. Abgesehen von den „klassischen“ Risikofaktoren sollte auch die Zahnstellung in Bezug auf das Alveolarfach berücksichtigt werden. Bei einer prominenten Stellung der Wurzel oder des gesamten Zahnes außerhalb des Alveolarfaches könnte eine Korrektur der Zahnfehlstellung vor der Rezessionsdeckung empfehlenswert sein. Abgesehen von vereinzelten Fallberichten gibt es jedoch kaum Studien zu dieser Fragestellung.

Eine retrospektive Auswertung an 20 Patienten zeigte nach Retroinklination von zuvor prominent stehenden Zähnen mit und ohne chirurgische Intervention einen signifikanten Rückgang der gingivalen Rezessionen [18]. Des Weiteren präsentierten Pini-Prato und Kollegen [23] in einem Fallbericht einen Patienten mit einem massiven Tiefbiss und einem traumatischen Einbiss bukkal der Unterkieferinzisivi. Dieser Einbiss wurde als hauptverantwortlich für die gingivalen Rezessionen (bis zu $7 \mathrm{~mm}$ ) erachtet; nach kieferorthopädischer Korrektur kam es zur vollständigen Heilung der gingivalen Rezessionen und eine zuvor noch geplante chirurgische Rezessionsdeckung war nicht mehr notwendig. In einem weiteren Fallbericht von Zucchelli et al. [24] wird ein mögliches Vorgehen bei einem Zahn mit gingivaler Rezession und Stellung außerhalb des Alveolarfaches beschrieben. Durch die kieferorthopädische Therapie wurde die Zahnfehlstellung korrigiert und im Anschluss erfolgte die Rezessionsdeckung mit ästhetisch ansprechendem Endergebnis.

Somit kann weder die Frage, ob die Rezessionsdeckung vor oder nach Kieferorthopädie erfolgen sollte, noch, ob es prognostisch für die Rezessionsdeckung vorteilhaft ist zunächst die Zahnfehlstellung zu korrigieren, mit der bestehenden Literatur eindeutig beantwortet werden. Damit ist einmal mehr bei dieser Thematik der Therapieentscheid subjektiv und nicht evidenz-basiert zu treffen. Als vorteilhaft an einer kieferorthopädischen Therapie vor Rezessionsdeckung sind in jedem Fall folgende Punkte anzusehen: erleichterte Mundhygiene, Positionierung des Zahnes innerhalb des Alveolarfaches, Reduktion der Weichgewebsspannung bei einer prominenten Wurzelstellung, Aufhebung eines okklusalen Traumas und/oder eines traumatischen Einbisses [21]. Des Weiteren sollte in den Therapieüberlegungen das Hauptanliegen des Patienten beachtet werden - Kieferorthopädie oder Rezessionsdeckung?

Überlegungen bei dem primären Patientenwunsch „Kieferorthopädie“ ( $\bullet$ Abb. 3):

- Steht der Zahn außerhalb des Alveolarfaches?

- Erfolgt die geplante Bewegung zurück in Richtung Alveolarfach oder bleibt die Position des Zahnes in Bezug auf das Alveolarfach unverändert?

- Ist die Hygienefähigkeit bei der gingivalen Rezession gegeben?

- Liegen weitere Risikofaktoren für das Entstehen gingivaler Rezessionen vor?

Ist die Hygienefähigkeit gegeben und erfolgt die geplante Bewegung tendenziell zurück ins Alveolarfach, kann unter regelmäßigen Kontrollen zunächst mit der kieferorthopädischen Therapie begonnen werden und die Rezessionsdeckung erst nach Korrektur der Zahnfehlstellung geplant werden [24]. Ist die Hygienefähigkeit nicht gegeben (z.B. ausgedehnte Miller-Klasse II mit nicht befestigtem marginalen Gingivarand), sollte eine Korrektur vorab angedacht werden, um auch die Zahnbewegung nicht im entzündeten Areal durchzuführen; nicht zuletzt aufgrund der zusätzlich erschwerten Hygienesituation während der kieferorthopädischen Behandlung. Ebenso kann eine Rezessionsdeckung vorab beim gleichzeitigen Vorliegen anderer Risikofaktoren zur Vorbeugung einer Progression der gingivalen Rezession angedacht werden ( $\odot$ Abb. 4). Erfolgt die Rezessionsdeckung vor der Kieferorthopädie, ist zu bedenken, dass dadurch lediglich 


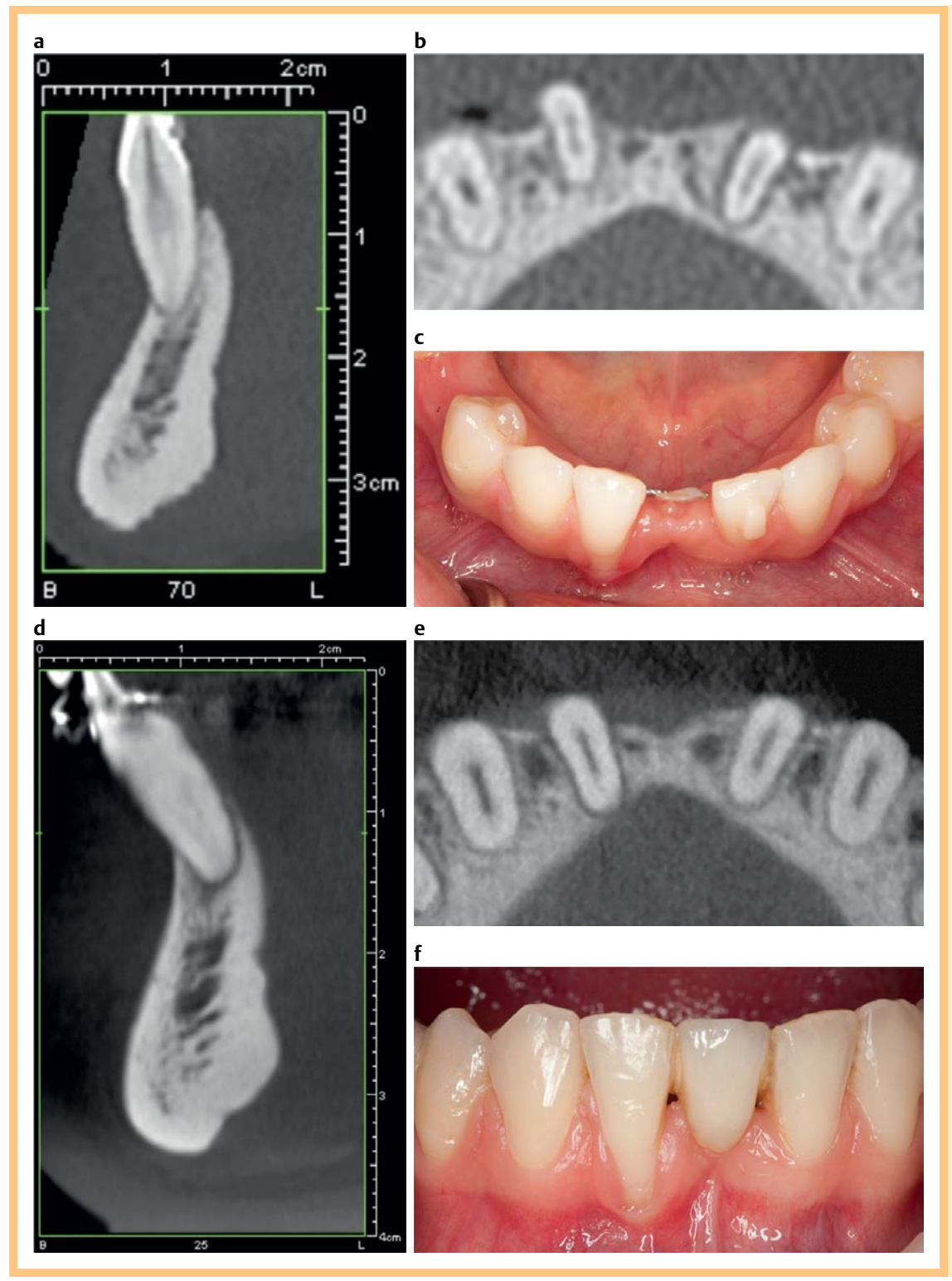

Abb. 3 a-c Patientin wird nach kieferorthopädischer Vorbehandlung vorstellig. Es liegen 3 Nichtanlagen $(31,41,45)$ und ein knöchernes Defizit b im Bereich der fehlenden mittleren Unterkieferinzisivi vor. Vor erneuter kieferorthopädischer Behandlung steht Zahn 42 in einer Position außerhalb des Alveolarfaches a mit einer Dehiszenz der bukkalen Knochenlamelle bis fast auf Apexniveau. d-f Zahn 42 wurde in das Alveolarfach zurückbewegt $\mathbf{d}$, wobei hier das knöcherne Defizit im Bereich der Unterkieferinzisivi deutlich wird; e der Alveolarkamm ist zu schmal für den gesamten Umfang der Wurzeln von 32 und 42. Die Patientin wollte nach Abschluss der Versorgung (KFO, Implantat 45, Klebebrücke 42-32) vorerst keinen weiteren Eingriff zur Rezessionsdeckung an Zahn 42. Aufgrund der gegebenen Hygienefähigkeit, Entzündungsfreiheit und einem stabilen, befestigten marginalen Gingivarand besteht auch keine medizinische Indikation zur Rezessionsdeckung. (Kieferorthopädie:

Dr. Michael Bertl, Fachbereich für Kieferorthopädie, Universitätszahnklinik Wien; Prothetik:

Dr. Lana Zupancic Cepic, Fachbereich für Prothetik, Universitätszahnklinik Wien).
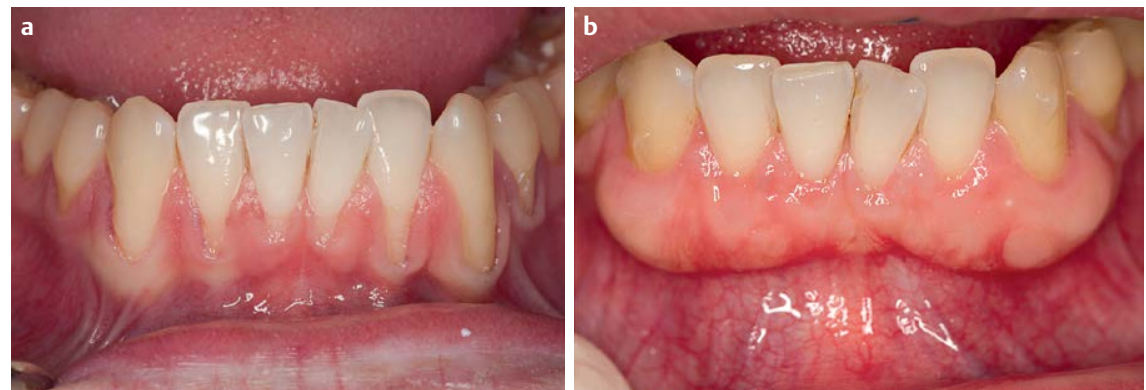

Abb. 4 a Patient mit gingivalen Rezessionen bis zu $6 \mathrm{~mm}$ und einem schmalen Band an keratinisierter Gingiva $(<1 \mathrm{~mm})$ im Bereich der Unterkieferfront. Um einer Progression während der kieferorthopädischen Behandlung vorzubeugen, erfolgte eine Deckung der gingivalen Rezessionen vor Beginn der Kieferorthopädie (modifizierte Tunnelierungstechnik mit autologem Bindegewebstransplantat). b Deutliche Zunahme der bukko-lingualen Weichgewebsdimension und vollständige Deckung der gingivalen Rezessionen (33-43; 1 Jahr nach dem chirurgischen Eingriff und bei Beginn der kieferorthopädischen Behandlung). (Kieferorthopädie: Dr. Frank Falkensammer, Fachbereich für Kieferorthopädie, Universitätszahnklinik Wien).

der Weichgewebsanteil und nicht das ebenso fehlende Hartgewebe rekonstruiert wird. Deswegen sollte auch nach einer Rezessionsdeckung eine Expansion des Zahnbogens oder eine Bewegung tendenziell aus dem Alveolarfach hinaus wenn überhaupt möglichst gering sein.
Überlegungen bei dem primären Patientenwunsch „Rezessionsdeckung“ ( 0 Abb. 5)

- Steht der Zahn außerhalb des Alveolarfaches?

- Ist eine Zahnfehlstellung (z.B. traumatischer Einbiss) als ursächlich für die gingivale Rezession anzusehen? 

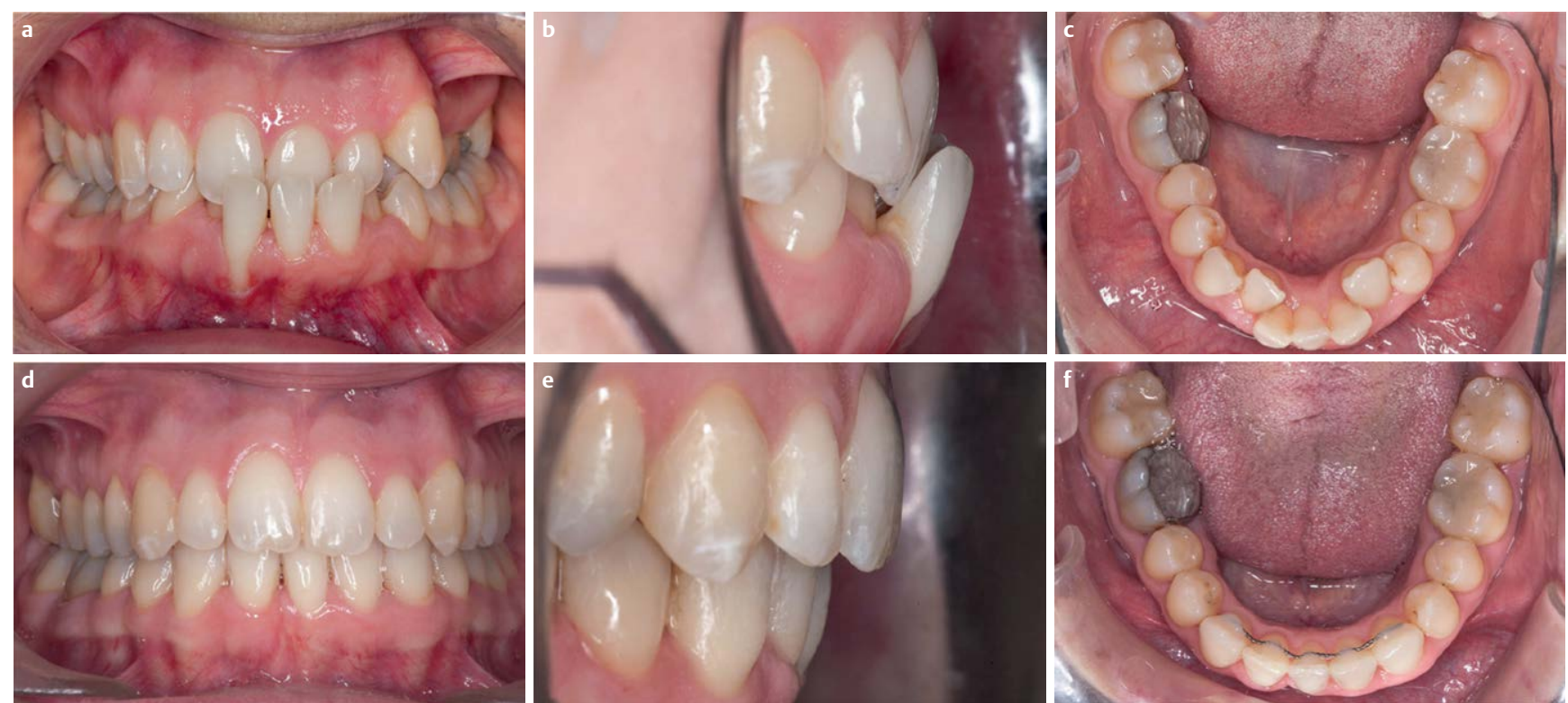

Abb. 5 a-c Patientin mit dem primären Wunsch zur Rezessionsdeckung an Zahn 41. Nach einer Beratung stimmte die Patientin einer kieferorthopädischen Behandlung zu. Aufgrund des deutlichen Engstandes wurde entschieden 41 zu extrahieren. d-f Ergebnis nach kieferorthopädischer Behandlung. Nach Extraktion von 41 war bei dieser Patientin kein parodontal-chirurgischer Eingriff mehr notwendig. (Kieferorthopädie: Dr. Michael Bertl, Fachbereich für Kieferorthopädie, Universitätszahnklinik Wien).

- Liegt aufgrund der Zahnfehlstellung eine erschwerte Hygienesituation vor?

Bei Vorliegen einer vermutlich ursächlichen Zahnfehlstellung sollte mit dem Patienten die Möglichkeit einer kieferorthopädischen Therapie besprochen werden. Dadurch wird vermutlich das Ergebnis der Rezessionsdeckung verbessert und einem Rezidiv entgegengewirkt. $\mathrm{Zu}$ besprechen ist hierbei natürlich auch der erhöhte Therapie- und Kostenaufwand für den Patienten.

\section{Zusammenfassung}

- Aufgrund der nicht ausreichenden Studienlage ist der Therapieentscheid, ob die Rezessionsdeckung vor oder nach der kieferorthopädischen Behandlung erfolgen sollte, subjektiv zu treffen.

- Die Bewegungsrichtung während der kieferorthopädischen Behandlung sowie das Vorliegen anderer Risikofaktoren sollten im Therapieentscheid beachtet werden.

- Bei einer Zahnfehlstellung sollte vor Rezessionsdeckung mit dem Patienten die Möglichkeit einer kieferorthopädischen Behandlung zur Korrektur der Fehlstellung besprochen werden.

- Randomisierte, kontrollierte Studien sind zu dieser Thematik notwendig.

\section{Schlussfolgerung \\ $\nabla$}

Ob eine kieferorthopädische Behandlung als (mit)ursächlich für gingivale Rezessionen angesehen werden kann, wird nach wie vor kontrovers diskutiert; rezente Studien mit einer langen Nachverfolgung in der Retentionsphase wiesen jedoch durchwegs eine höhere Prävalenz an gingivalen Rezessionen nach kieferorthopädischer Behandlung auf. Des Weiteren kann von einem erhöhten Risiko für die Entstehung von gingivalen Rezessionen bei gleichzeitigem Vorliegen von „klassischen“ Risikofaktoren (dünner marginaler Gingivarand, dünner gingivaler Phänotyp, schmales Band an keratinisierter Gingiva, bereits bestehende knöcherne Dehiszenz, hohe Plaquewerte, traumatische Putztechnik, uvm.) ausgegangen werden und eine Zahnbewegung aus dem Alveolarfach hinaus sollte möglichst vermieden werden. Sowohl die Frage nach der Indikation zur „prophylaktischen“ Weichgewebsaugmentation vor Beginn der kieferorthopädischen Behandlung als auch nach dem richtigen Zeitpunkt für eine Deckung einer bereits bestehenden gingivalen Rezession (vor oder nach Kieferorthopädie) sind mit der bestehenden Literatur nicht eindeutig zu beantworten. Somit bleibt der Therapieentscheid subjektiv und nicht evidenz-basiert zu treffen und es besteht ein Bedarf an hochqualitativen, prospektiven und kontrollierten Langzeitstudien.

\section{Literatur}

1 Susin C, Haas AN, Oppermann RV et al. Gingival recession: epidemiology and risk indicators in a representative urban Brazilian population. J Periodontol 2004; 75: 1377-1386

2 Bertl K, Durstberger G, Ulm C. Von der Planung bis zur Deckung gingivaler Rezessionen. Stomatologie 2013; 110: 21-26

3 Wedrychowska-Szulc B, Syrynska M. Patient and parent motivation for orthodontic treatment - a questionnaire study. Eur J Orthod 2010; 32: $447-452$

4 Wennstrom JL. Mucogingival considerations in orthodontic treatment. Semin Orthod 1996; 2: 46-54

5 Boke F, Gazioglu C, Akkaya S et al. Relationship between orthodontic treatment and gingival health: A retrospective study. Eur J Dent 2014; 8: $373-380$

6 Yared KF, Zenobio EG, Pacheco W. Periodontal status of mandibular central incisors after orthodontic proclination in adults. Am J Orthod Dentofacial Orthop 2006; 130: 6.e1-6.e8

7 Renkema AM, Navratilova Z, Mazurova $K$ et al. Gingival labial recessions and the post-treatment proclination of mandibular incisors. Eur J Orthod 2014

8 Renkema AM, Fudalej PS, Renkema A et al. Gingival recessions and the change of inclination of mandibular incisors during orthodontic treatment. Eur J Orthod 2013; 35: 249-255

9 Joss-Vassalli I, Grebenstein C, Topouzelis $N$ et al. Orthodontic therapy and gingival recession: a systematic review. Orthod Craniofac Res 2010; 13: 127-141

10 Aziz T, Flores-Mir C. A systematic review of the association between appliance-induced labial movement of mandibular incisors and gingival recession. Aust Orthod J 2011; 27: 33-39 
11 Renkema AM, Fudalej PS, Renkema A et al. Development of labia gingival recessions in orthodontically treated patients. Am J Orthod Dentofacial Orthop 2013; 143: 206-212

12 Renkema AM, Fudalej PS, Renkema AA et al. Gingival labial recessions in orthodontically treated and untreated individuals: a case - control study. J Clin Periodontol 2013; 40: 631-637

13 Vasconcelos G, Kjellsen K, Preus $H$ et al. Prevalence and severity of vestibular recession in mandibular incisors after orthodontic treatment. Angle Orthod 2012; 82: 42-47

14 Baldi C, Pini-Prato G, Pagliaro U et al. Coronally advanced flap procedure for root coverage. Is flap thickness a relevant predictor to achieve root coverage? A 19-case series. J Periodontol 1999; 70: 1077-1084

15 Huang $L H$, Neiva RE, Wang HL. Factors affecting the outcomes of coronally advanced flap root coverage procedure. J Periodontol 2005; 76: $1729-1734$

16 Kloukos D, Eliades T, Sculean A et al. Indication and timing of soft tissue augmentation at maxillary and mandibular incisors in orthodontic patients. A systematic review. Eur J Orthod 2014; 36: 442-449

17 Maynard JGJ, Ochsenbein C. Mucogingival problems, prevalence and therapy in children. J Periodontol 1975; 46: 543-552

18 Ngan PW, Burch JG, Wei SH. Grafted and ungrafted labial gingival recession in pediatric orthodontic patients: effects of retraction and inflammation. Quintessence Int 1991; 22: 103-111
19 Chambrone L, Chambrone D, Pustiglioni FE et al. Can subepithelial connective tissue grafts be considered the gold standard procedure in the treatment of Miller Class I and II recession-type defects? J Dent 2008; 36: 659-671

20 Roccuzzo M, Bunino M, Needleman I et al. Periodontal plastic surgery for treatment of localized gingival recessions: a systematic review. J Clin Periodontol 2002; 29: (Suppl 3): 178-194 discussion 195

21 Johal A, Katsaros C, Kiliaridis $S$ et al. State of the science on controversial topics: orthodontic therapy and gingival recession (a report of the Angle Society of Europe 2013 meeting). Prog Orthod 2013; 14: 16

22 Kim DM, Neiva R. Periodontal soft tissue non-root coverage procedures: a systematic review from the AAP regeneration workshop. J Periodontol 2015; 86: S56-S72

23 Pini-Prato GP, Cozzani G, Magnani C et al. Healing of gingival recession following orthodontic treatment: a 30-year case report. Int J Periodontics Restorative Dent 2012; 32: 23-27

24 Zucchelli G, Parenti SI, Ghigi G et al. Combined orthodontic - mucogingival treatment of a deep post-orthodontic gingival recession. Eur J Esthet Dent 2012; 7: 266-280

25 Artun J, Krogstad O. Periodontal status of mandibular incisors following excessive proclination. A study in adults with surgically treated mandibular prognathism. Am J Orthod Dentofacial Orthop 1987; 91: 225-232 